\title{
Throughput of Nonbinary Type-II Hybrid ARQ with Adaptive Modulation
}

\author{
Lijun Zhang ${ }^{1}$ \\ Dept. of Electronic Engineering \\ Tsinghua Univ. \\ Beijing, 100084, CHINA
}

\author{
Victor O. K. Li \\ Dept. of Electrical and Electronic Engineering \\ Univ.of Hong Kong \\ Pokfulam Road, Hong Kong, CHINA \\ e-mail: vli@eee.hku.hk
}

\author{
Zhigang Cao \\ Dept. of Electronic Engineering \\ Tsinghua Univ. \\ Beijing, 100084, CHINA \\ e-mail: czg-dee@tsinghua.edu.cn
}

\section{INTRODUCTION}

Hybrid Automatic-Repeat-reQuest (ARQ) combines the advantages of ARQ and Forward-Error-Control (FEC), and can achieve good throughput with very low bit error rate (BER) [1]. In this paper, nonbinary HARQ is proposed and analyzed in terms of throughput. Nonbinary HARQ is also applied in a cell with adaptive modulation and the average throughput is obtained.

\section{NONBINARY TYPE-II HARQ}

Type-II HARQ uses FEC for error correction, but only retransmits the necessary part, such as the information or parity check part, rather than the whole packet, as in type-I. The most widely used protocol is to combine 1/2-rate invertible codes with ARQ [2]. With invertible codes, all the information in the packet may be retrieved from the error-free parity check part.

In our nonbinary HARQ, half rate shortened Reed-Solomon (RS) code is used in the FEC subsystem instead of binary BCH codes. The latter has been used in Lin and Yu's original type-II HARQ [2]. The merits of using RS codes are obvious. For one thing, RS codes are suited to correct long-burst errors, which degrade transmission quality in wireless communications; for another, they belong to the class of Maximum-Distance-Separable (MDS) codes, and allows flexibility in adjusting the coding rate. In addition, RS codes can be easily combined with such bandwidth efficient modulation schemes as MPSK, or QAM, allowing the full use of the precious spectrum resource.

The throughput of nonbinary HARQ can be derived similarly as in [2]. Due to space limitations, the results will not be presented here.

\section{HARQ WITH ADAPTIVE MODULATION}

Assume that nonbinary HARQ is applied to a cell, which is divided into two circular regions, $A_{1}$ and $A_{2}$, with the transmission antenna at the center. Mobile terminals (MTs) in $A_{1}$ are near the antenna, and encounter better channel states in comparison with those MTs in $A_{2}$. Accordingly, high throughput HARQ should be used in $A_{1}$, and relatively low throughput HARQ, in $A_{2}$. HARQ schemes with different throughputs can be implemented by changing the size of the modulation constellation. Two MPSK schemes, QPSK and 8PSK, are considered in our research, for their wide use in current communication system. The two modulation schemes are switched according to the average SNR of the received signal, viz., the distance from the base station. 8PSK and QPSK are adopted in the near and far regions, respectively.

To evaluate different partitioning of a cell, we define the modulation assignment index

$$
f=\frac{\sharp \text { of MTs in } A_{1}}{\text { total } \sharp \text { of MTs in the cell }} \text {. }
$$

\footnotetext{
${ }^{1}$ This research is supported in part by the National Natural Science Foundation of China (No.6001161943)/Research Grants Council of Hong Kong Joint Research Scheme under Grant No. N_HKU 709/00.
}

Assume the mean throughput of the user at distance $r$ from the antenna to be $\eta_{M P S K \mid r}$, and the $p d f$ of $r$ to be $p(r)$, we average $\eta_{M P S K \mid r}$ over the whole cell, and get $\eta=\int_{A_{1}} \eta_{8 P S K \mid r} p(r) d r+$ $\int_{A_{2}} \eta_{Q P S K \mid r} p(r) d r$

\section{NUMERICAL RESULTS}

Two nonbinary type-II HARQs are considered, RS $(60,30)$ code with 8PSK and QPSK, respectively. Let the roundtrip delay be $33 \mathrm{~ms}$, data rate be $2 \mathrm{Mbps}$, radius of the cell be $1000 \mathrm{~m}$, and operation frequency be $900 \mathrm{MHz}$. Slow, flat, Rician fading with a Rician parameter of $5 \mathrm{~dB}$ is included in the calculation.

Figure 1 shows a cluster of throughput curves with a parameter of $\gamma_{d 0}$, SNR at reference distance $d_{0}$ from the antenna. It is easily observed that an optimum $f$ exists, corresponding to the maximal value of the average throughput. From the calculation of the average throughput, we conclude that the optimal threshold of SNR is where the throughput curves of QPSK and 8PSK coincide.

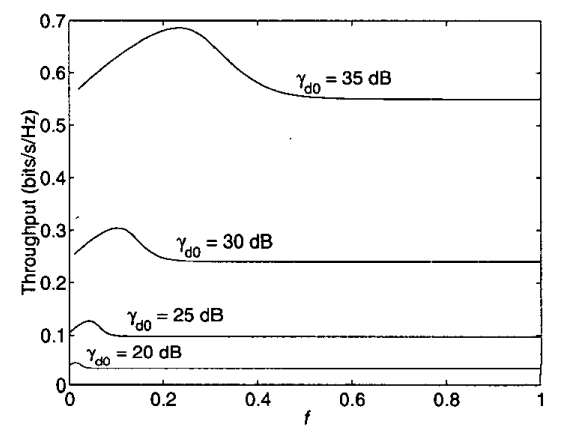

Figure 1: Throughput of HARQs with adaptive modulation as a function of $f$ with error-correction capability $t=5$ of shortened RS $(60,30)$ code.

Furthermore, type-II HARQ with adaptive modulation can achieve much higher throughput compared to the non-adaptive ones. Considering the case of $\gamma_{d_{0}}=30 \mathrm{~dB}$, the maximal throughput exceeds $0.30 \mathrm{bits} / \mathrm{s} / \mathrm{Hz}$, while the corresponding throughputs of fixed 8PSK $(f=1)$ and QPSK $(f=0)$ are 0.24 and $0.25 \mathrm{bits} / \mathrm{s} / \mathrm{Hz}$, respectively. Over $25 \%$ throughput gain can be achieved with the adaptive scheme.

\section{REFERENCES}

[1] S. Lin, and D. J. Costello Jr., Error Control Coding: Fundamentals and Applications, Englewood Cliffs, NJ: Prentice-Hall, 1983.

[2] S. Lin, and P. S. Yu, "A hybrid ARQ scheme with parity retransmission for error control of satellite channels," IEEE Trans. Commun., vol. COM30, pp. 1701-1719, Jul. 1982. 